\title{
GAUDIN HAMILTONIANS GENERATE THE BETHE ALGEBRA OF A TENSOR POWER OF THE VECTOR REPRESENTATION OF $\mathfrak{g l}_{N}$
}

\author{
E. MUKHIN, V. TARASOV, AND A. VARCHENKO
}

To Ludwig Dmitrievich Faddeev on his 75 th birthday

\begin{abstract}
It is shown that the Gaudin Hamiltonians $H_{1}, \ldots, H_{n}$ generate the Bethe algebra of the $n$-fold tensor power of the vector representation of $\mathfrak{g l}_{N}$. Surprisingly, the formula for the generators of the Bethe algebra in terms of the Gaudin Hamiltonians does not depend on $N$. Moreover, this formula coincides with Wilson's formula for the stationary Baker-Akhiezer function on the adelic Grassmannian.
\end{abstract}

\section{$\S 1$. INTRODUCTION}

The Gaudin model describes a completely integrable quantum spin chain G1, G2. We consider the Gaudin model associated with the Lie algebra $\mathfrak{g l}_{N}$. Denote by $L_{\boldsymbol{\lambda}}$ the irreducible finite-dimensional $\mathfrak{g l}_{N}$-module with highest weight $\boldsymbol{\lambda}$. Consider a tensor product $\bigotimes_{a=1}^{n} L_{\boldsymbol{\lambda}^{(a)}}$ of such modules and two sequences of complex numbers: $K_{1}, \ldots, K_{N}$ and $z_{1}, \ldots, z_{n}$. Assume that the numbers $z_{1}, \ldots, z_{n}$ are distinct. The Hamiltonians of the Gaudin model are mutually commuting operators $H_{1}, \ldots, H_{n}$ acting on the space $\bigotimes_{a=1}^{n} L_{\boldsymbol{\lambda}^{(a)}}$,

$$
H_{a}=\sum_{i=1}^{N} K_{i} e_{i i}^{(a)}+\sum_{i, j=1}^{N} \sum_{b \neq a} \frac{e_{i j}^{(a)} e_{j i}^{(b)}}{z_{a}-z_{b}},
$$

where the $e_{i j}$ are the standard generators of $\mathfrak{g l}_{N}$, and $e_{i j}^{(a)}$ is the image of $1^{\otimes(a-1)} \otimes e_{i j} \otimes$ $1^{\otimes(n-a)}$.

One of the main problems in the Gaudin model is to find eigenvalues and joint eigenvectors of the operators $H_{1}, \ldots, H_{n}$; see [B, RV, MTV1. The Gaudin Hamiltonians appear also as the right-hand sides of the Knizhnik-Zamolodchikov equations; see [SV, RV, FFR, FMTV].

It was realized a long time ago that there are additional interesting operators commuting with the operators $H_{1}, \ldots, H_{n}$; see for example [KS, FFR. Those operators are called the higher Gaudin Hamiltonians. To distinguish the operators $H_{1}, \ldots, H_{n}$, we will call them the classical Gaudin Hamiltonians. The algebra generated by all of the classical and higher Gaudin Hamiltonians is called the Bethe algebra. A useful formula for generators of the Bethe algebra was suggested in [T]; see also [MTV1, CT.

2010 Mathematics Subject Classification. Primary 82C23, 81Q12.

Key words and phrases. Gaudin model, Bethe algebra, Calogero-Moser space.

E. Mukhin was supported in part by NSF grant DMS-0900984.

V. Tarasov was supported by NSF grant DMS-0901616.

A. Varchenko was supported by NSF grant DMS-0555327. 
In general, the Bethe algebra is larger than its subalgebra generated by the classical Gaudin Hamiltonians. Nevertheless, in this paper we show that if all factors of the tensor product $\bigotimes_{a=1}^{n} L_{\boldsymbol{\lambda}^{(a)}}$ are the standard vector representations of $\mathfrak{g l}_{N}$, then the classical Gaudin Hamiltonians generate the entire Bethe algebra. It is a surprising fact, because every tensor product of polynomial $\mathfrak{g l}_{N}$-modules is a submodule of a tensor power of the vector representation, and one may expect that the Bethe algebra of a tensor power of the vector representation is as general as the Bethe algebra of a tensor product of arbitrary representations. Another surprising fact is that our formula for the elements of the Bethe algebra in terms of the classical Gaudin Hamiltonians does not depend on $N$; see Theorem 3.2. The third surprise is that our formula is nothing else but Wilson's formula for the stationary Baker-Akhiezer function on the adelic Grassmannian Wi].

Our theorem can be used to study the higher Gaudin Hamiltonians as functions of the classical Hamiltonians (or as limits of functions of the classical Gaudin Hamiltonians). Much more is known about the classical Hamiltonians than about the higher Hamiltonians.

Our proof of Theorem 3.2 is not elementary. We use the fact that the Bethe algebra is preserved under the $\left(\mathfrak{g l}_{N}, \mathfrak{g l}_{n}\right)$-duality and the completeness of the Bethe ansatz for a tensor product of vector representations and generic $K_{1}, \ldots, K_{N}, z_{1}, \ldots, z_{n}$.

\section{$\S 2$. Bethe Algebra}

2.1. Lie algebras $\mathfrak{g l}_{N}$ and $\mathfrak{g l}_{N}[t]$. Let $e_{i j}, i, j=1, \ldots, N$, be the standard generators of the Lie algebra $\mathfrak{g l}_{N}$ satisfying the relations $\left[e_{i j}, e_{s k}\right]=\delta_{j s} e_{i k}-\delta_{i k} e_{s j}$. Let $\mathfrak{h} \subset \mathfrak{g l}_{N}$ be the Cartan subalgebra generated by $e_{i i}, i=1, \ldots, N$.

We denote by $V=\bigoplus_{i=1}^{N} \mathbb{C} v_{i}$ the standard $N$-dimensional vector representation of $\mathfrak{g l}_{N}: e_{i j} v_{j}=v_{i}$ and $e_{i j} v_{k}=0$ for $j \neq k$.

Let $M$ be a $\mathfrak{g l}_{N}$-module. A vector $v \in M$ is said to be singular if $e_{i j} v=0$ for $1 \leq i<j \leq N$. We denote by $M^{\text {sing }}$ the subspace of all singular vectors in $M$.

Let $\mathfrak{g l}_{N}[t]=\mathfrak{g l}_{N} \otimes \mathbb{C}[t]$ be the complex Lie algebra of $\mathfrak{g l}_{N}$-valued polynomials with the pointwise commutator. For $g \in \mathfrak{g l}_{N}$, we set $g(u)=\sum_{s=0}^{\infty}\left(g \otimes t^{s}\right) u^{-s-1}$.

We identify $\mathfrak{g l}_{N}$ with the subalgebra $\mathfrak{g l}_{N} \otimes 1$ of constant polynomials in $\mathfrak{g l}_{N}[t]$. Hence, any $\mathfrak{g l}_{N}[t]$-module has a canonical structure of a $\mathfrak{g l}_{N}$-module.

For each $a \in \mathbb{C}$, there exists an automorphism $\rho_{a}$ of $\mathfrak{g l}_{N}[t], \rho_{a}: g(u) \mapsto g(u-a)$. Given a $\mathfrak{g l}_{N}[t]$-module $M$, we denote by $M(a)$ the pullback of $M$ through the automorphism $\rho_{a}$. As $\mathfrak{g l}_{N}$-modules, $M$ and $M(a)$ are isomorphic by the identity map.

We have the evaluation homomorphism, $\mathfrak{g l}_{N}[t] \rightarrow \mathfrak{g l}_{N}, g(u) \mapsto g u^{-1}$. Its restriction to the subalgebra $\mathfrak{g l}_{N} \subset \mathfrak{g l}_{N}[t]$ is the identity map. For any $\mathfrak{g l}_{N}$-module $M$, we denote by the same letter the $\mathfrak{g l}_{N}[t]$-module obtained by pulling $M$ back through the evaluation homomorphism.

2.2. Bethe algebra. Given an $N \times N$-matrix $A$ with possibly noncommuting entries $a_{i j}$, we define its row determinant to be

$$
\operatorname{rdet} A=\sum_{\sigma \in S_{N}}(-1)^{\sigma} a_{1 \sigma(1)} a_{2 \sigma(2)} \ldots a_{N \sigma(N)} .
$$

Let $K_{1}, \ldots, K_{N}$ be a sequence of complex numbers. Let $\partial_{u}$ be the operator of differentiation in the variable $u$. Define the universal differential operator $\mathcal{D}^{K}$ by the formula

$$
\mathcal{D}^{K}=\operatorname{rdet}\left(\begin{array}{cccc}
\partial_{u}-K_{1}-e_{11}(u) & -e_{21}(u) & \ldots & -e_{N 1}(u) \\
-e_{12}(u) & \partial_{u}-K_{2}-e_{22}(u) & \ldots & -e_{N 2}(u) \\
\ldots & \ldots & \ldots & \ldots \\
-e_{1 N}(u) & -e_{2 N}(u) & \ldots & \partial_{u}-K_{N}-e_{N N}(u)
\end{array}\right) .
$$


This is a differential operator in $u$, whose coefficients are formal power series in $u^{-1}$ with coefficients in $U\left(\mathfrak{g l}_{N}[t]\right)$,

$$
\mathcal{D}^{K}=\partial_{u}^{N}+\sum_{i=1}^{N} B_{i}^{K}(u) \partial_{u}^{N-i}, \quad B_{i}^{K}(u)=\sum_{j=0}^{\infty} B_{i j}^{K} u^{-j},
$$

and $B_{i j}^{K} \in U\left(\mathfrak{g l}_{N}[t]\right), i=1, \ldots, N, j \in \mathbb{Z}_{\geq 0}$. We have

$$
\partial_{u}^{N}+\sum_{i=1}^{N} B_{i 0}^{K} \partial_{u}^{N-i}=\prod_{i=1}^{N}\left(\partial_{u}-K_{i}\right) .
$$

The unital subalgebra of $U\left(\mathfrak{g l}_{N}[t]\right)$ generated by $B_{i j}^{K}, i=1, \ldots, N, j \in \mathbb{Z}_{>0}$, is called the Bethe algebra and is denoted by $\mathcal{B}^{K}$.

By T, MTV1, CT, the algebra $\mathcal{B}^{K}$ is commutative, and $\mathcal{B}^{K}$ commutes with the subalgebra $U(\mathfrak{h}) \subset U\left(\mathfrak{g l}_{N}[t]\right)$. If all $K_{1}, \ldots, K_{N}$ coincide, then $\mathcal{B}^{K}$ commutes with the subalgebra $U\left(\mathfrak{g l}_{N}\right) \subset U\left(\mathfrak{g l}_{N}[t]\right)$.

As a subalgebra of $U\left(\mathfrak{g l}_{N}[t]\right)$, the algebra $\mathcal{B}^{K}$ acts on any $\mathfrak{g l}_{N}[t]$-module $M$. Since $\mathcal{B}^{K}$ commutes with $U(\mathfrak{h})$, it preserves the weight subspaces of $M$. If all $K_{1}, \ldots, K_{N}$ coincide, then $\mathcal{B}^{K}$ preserves the subspace $M^{\text {sing }}$ of singular vectors. If $L$ is a $\mathcal{B}^{K}$-module, then the image of $\mathcal{B}^{K}$ in $\operatorname{End}(L)$ is called the Bethe algebra of $L$.

For our purposes it is convenient to consider another set of generators of the Bethe algebra $\mathcal{B}^{K}$, defined as follows. Let $x$ be a new variable, and let

$$
\begin{aligned}
\Psi^{K}(u, x) & =\left(x^{N}+\sum_{i=1}^{N} B_{i}^{K}(u) x^{N-i}\right) \prod_{i=1}^{N} \frac{1}{x-K_{i}} \\
& =1+\sum_{i=1}^{\infty} \Psi_{i}^{K}(u) x^{-i} .
\end{aligned}
$$

The series $\Psi_{i}^{K}(u), i \in \mathbb{Z}_{>0}$, are linear combinations of the series $B_{i}^{K}(u), i=1, \ldots, N$, and vice versa. Write

$$
\Psi_{i}^{K}(u)=\sum_{j=1}^{\infty} \Psi_{i j}^{K} u^{-j}
$$

Then $\Psi_{i j}^{K}, i, j \in \mathbb{Z}_{>0}$, is a new set of generators of the Bethe algebra $\mathcal{B}^{K}$.

\section{§3. Classical Gaudin Hamiltonians on $\bigotimes_{a=1}^{n} V\left(z_{a}\right)$}

Recall that $V$ is the vector representation of the Lie algebra $\mathfrak{g l}_{N}$. Consider the tensor product $\bigotimes_{a=1}^{n} V\left(z_{a}\right)$ of evaluation $\mathfrak{g l}_{N}[t]$-modules. The series $e_{i j}(u)$ acts on $\bigotimes_{a=1}^{n} V\left(z_{a}\right)$ as $\sum_{a=1}^{n} e_{i j}^{(a)}\left(u-z_{a}\right)^{-1}$, where $e_{i j}^{(a)}$ is the image of $1^{\otimes(a-1)} \otimes e_{i j} \otimes 1^{\otimes(n-a)} \in\left(U\left(\mathfrak{g l}_{N}\right)\right)^{\otimes n}$. We denote by $B_{i j}, \Psi_{i j} \in \operatorname{End}\left(V^{\otimes n}\right)$ the images of the elements $B_{i j}^{K}, \Psi_{i j}^{K} \in U\left(\mathfrak{g l}_{N}[t]\right)$. Set

$$
\begin{aligned}
& B_{i}(u)=\sum_{j=0}^{\infty} B_{i j} u^{-j}, \quad \mathcal{D}=\partial_{u}^{N}+\sum_{i=1}^{N} B_{i}(u) \partial_{u}^{N-i}, \\
& \Psi_{i}(u)=\sum_{j=1}^{\infty} \Psi_{i j} u^{-j}, \quad \Psi(u, x)=1+\sum_{i=1}^{\infty} \Psi_{i}(u) x^{-i} .
\end{aligned}
$$

The series $B_{i}(u), \Psi_{i}(u)$ sum up to rational functions of $u$ with values in $\operatorname{End}\left(V^{\otimes n}\right)$. Set in addition

$$
\Psi_{\dagger}(x)=-\sum_{i=1}^{\infty} \Psi_{i 1} x^{-i}
$$


Lemma 3.1. We have

$$
\Psi_{1}(u)=-\sum_{a=1}^{n} \frac{1}{u-z_{a}}, \quad \Psi_{2}(u)=\sum_{a=1}^{n} \frac{1}{u-z_{a}}\left(-H_{a}+\sum_{b \neq a} \frac{1}{z_{a}-z_{b}}\right),
$$

where

$$
H_{a}=\sum_{i=1}^{N} K_{i} e_{i i}^{(a)}+\sum_{i, j=1}^{N} \sum_{b \neq a} \frac{e_{i j}^{(a)} e_{j i}^{(b)}}{z_{a}-z_{b}}
$$

are the classical Gaudin Hamiltonians (1.1), and

$$
\Psi_{\dagger}(x)=\sum_{i=1}^{N} \sum_{a=1}^{n} \frac{e_{i i}^{(a)}}{x-K_{i}} .
$$

Proof. The claim is straightforward. See also formula (8.5) and Appendix B in [MTV1].

To formulate our main result, we introduce a diagonal matrix

$$
Z=\operatorname{diag}\left(z_{1}, \ldots, z_{n}\right)
$$

and a matrix

$$
Q=\left(\begin{array}{ccccc}
h_{1} & \frac{1}{z_{2}-z_{1}} & \frac{1}{z_{3}-z_{1}} & \ldots & \frac{1}{z_{n}-z_{1}} \\
\frac{1}{z_{1}-z_{2}} & h_{2} & \frac{1}{z_{3}-z_{2}} & \ldots & \frac{1}{z_{n}-z_{2}} \\
\ldots & \ldots & \ldots & \ldots & \ldots \\
\frac{1}{z_{1}-z_{n}} & \frac{1}{z_{2}-z_{n}} & \frac{1}{z_{3}-z_{n}} & \ldots & h_{n}
\end{array}\right)
$$

depending on new variables $h_{1}, \ldots, h_{n}$. Set

$$
\begin{aligned}
\psi\left(u, x, z_{1}, \ldots, z_{n}, h_{1}, \ldots, h_{n}\right) & =\operatorname{det}\left(1-(u-Z)^{-1}(x-Q)^{-1}\right), \\
\phi\left(x, z_{1}, \ldots, z_{n}, h_{1}, \ldots, h_{n}\right) & =\operatorname{det}(x-Q), \\
\psi_{\dagger}\left(x, z_{1}, \ldots, z_{n}, h_{1}, \ldots, h_{n}\right) & =\operatorname{tr}\left((x-Q)^{-1}\right) .
\end{aligned}
$$

Theorem 3.2. The Bethe algebra of $\bigotimes_{a=1}^{n} V\left(z_{a}\right)$ is generated by the classical Gaudin Hamiltonians $H_{1}, \ldots, H_{n}$. More precisely,

$$
\Psi(u, x)=\psi\left(u, x, z_{1}, \ldots, z_{n}, H_{1}, \ldots, H_{n}\right) .
$$

In particular,

$$
\psi_{\dagger}\left(x, z_{1}, \ldots, z_{n}, H_{1}, \ldots, H_{n}\right)=\sum_{i=1}^{N} \sum_{a=1}^{n} \frac{e_{i i}^{(a)}}{x-K_{i}} .
$$

Remark. Since $\operatorname{tr}\left((x-Q)^{-1}\right)=\partial_{x} \log (\operatorname{det}(x-Q))$, formula (3.7) can be written as

$$
\phi\left(x, z_{1}, \ldots, z_{n}, H_{1}, \ldots, H_{n}\right)=\prod_{i=1}^{N}\left(x-K_{i}\right)^{\sum_{a=1}^{n} e_{i i}^{(a)}} .
$$

Remark. The matrix $[Q, Z]+1$ has rank one. For every distinct $z_{1}, \ldots, z_{n}$ and every $h_{1}, \ldots, h_{n}$, the pair $(Q, Z)$ determines a point of the $n$th Calogero-Moser space, hence, a point of the adelic Grassmannian. The function $e^{u x} \psi\left(u, x, z_{1}, \ldots, z_{n}, h_{1}, \ldots, h_{n}\right)$ is the 
stationary Baker-Akhiezer function of that point; see $\S 3$ in Wi. Theorem 3.2 says that the coefficients $\psi_{i j}\left(z_{1}, \ldots, z_{n}, H_{1}, \ldots, H_{n}\right)$ of the stationary Baker-Akhiezer function,

$$
\begin{aligned}
e^{u x} \psi & \left(u, x, z_{1}, \ldots, z_{n}, H_{1}, \ldots, H_{n}\right) \\
& =e^{u x}\left(1+\sum_{i, j=1}^{\infty} \psi_{i j}\left(z_{1}, \ldots, z_{n}, H_{1}, \ldots, H_{n}\right) u^{-j} x^{-i}\right),
\end{aligned}
$$

generate the Bethe algebra of $\bigotimes_{a=1}^{n} V\left(z_{a}\right)$. More remarks on this subject can be found in $\$ 5$,

Corollary 3.3. For distinct real $K_{1}, \ldots, K_{N}$, and distinct real $z_{1}, \ldots, z_{n}$, the joint spectrum of the classical Gaudin Hamiltonians $H_{1}, \ldots, H_{n}$ acting on $\bigotimes_{a=1}^{n} V\left(z_{a}\right)$ is simple. That is, the classical Gaudin Hamiltonians have a joint eigenbasis, and for any two vectors of the eigenbasis at least one of the classical Gaudin Hamiltonians has different eigenvalues for those vectors.

Proof. By [MTV5], for distinct real $K_{1}, \ldots, K_{N}$, and distinct real $z_{1}, \ldots, z_{n}$, the Bethe algebra of $\bigotimes_{a=1}^{n} V\left(z_{a}\right)$ has simple spectrum. Therefore, the classical Gaudin Hamiltonians have simple spectrum by Theorem 3.2 .

Corollary 3.4. If $K_{1}, \ldots, K_{N}$ coincide, and $z_{1}, \ldots, z_{n}$ are distinct and real, then the joint spectrum of classical Gaudin Hamiltonians $H_{1}, \ldots, H_{n}$ acting on $\left(\bigotimes_{a=1}^{n} V\left(z_{a}\right)\right)^{\text {sing }}$ is simple.

Proof. By [MTV3], if $K_{i}=0$ for all $i=1, \ldots, N$, and $z_{1}, \ldots, z_{n}$ are real and distinct, then the Bethe algebra of $\left(\bigotimes_{a=1}^{n} V\left(z_{a}\right)\right)^{\text {sing }}$ has simple spectrum. Therefore, the classical Gaudin Hamiltonians acting on $\left(\bigotimes_{a=1}^{n} V\left(z_{a}\right)\right)^{\text {sing }}$ have simple spectrum by Theorem 3.2 . The case of nonzero coinciding $K_{1}, \ldots, K_{N}$ follows from the case of zero $K_{1}, \ldots, K_{N}$, because $\sum_{i=1}^{N} e_{i i}^{(a)}=1$ for all $a=1, \ldots, n$; see (3.3).

\section{§4. Proof of Theorem 3.2}

4.1. Preliminary lemmas. For functions $f_{1}(x), \ldots, f_{m}(x)$ of one variable, denote by

$$
\mathrm{Wr}\left[f_{1}, \ldots, f_{m}\right]=\operatorname{det}\left(\begin{array}{cccc}
f_{1} & f_{1}^{\prime} & \ldots & f_{1}^{(m-1)} \\
f_{2} & f_{2}^{\prime} & \ldots & f_{2}^{(m-1)} \\
\ldots & \ldots & \ldots & \ldots \\
f_{m} & f_{m}^{\prime} & \ldots & f_{m}^{(m-1)}
\end{array}\right)
$$

the Wronskian of $f_{1}(x), \ldots, f_{m}(x)$. Set $\Delta=\prod_{1 \leq a<b \leq n}\left(z_{b}-z_{a}\right)$,

$$
P(u)=\prod_{a=1}^{n}\left(u-z_{a}\right)
$$

and

$$
P_{a}(u)=\prod_{b \neq a} \frac{u-z_{b}}{z_{a}-z_{b}}, \quad a=1, \ldots, n .
$$

Let $f_{a}(x)=\left(x+\mu_{a}\right) e^{z_{a} x}, a=1, \ldots, n$, where $\mu_{1}, \ldots, \mu_{n}$ are new variables. Set

$$
\begin{aligned}
W(u, x) & =e^{-u x-\sum_{a=1}^{n} z_{a} x} \operatorname{Wr}\left[f_{1}(x), \ldots, f_{n}(x), e^{u x}\right] \\
& =W_{0}(x)\left(u^{n}+\sum_{a=1}^{n} C_{a}(x) u^{n-a}\right) .
\end{aligned}
$$

Clearly, $W_{0}(x)=e^{-\sum_{a=1}^{n} z_{a} x} \operatorname{Wr}\left[f_{1}(x), \ldots, f_{n}(x)\right]$. 
Lemma 4.1. Let $h_{a}=-\mu_{a}-\sum_{b \neq a} \frac{1}{z_{a}-z_{b}}, a=1, \ldots, n$. Then

$$
W(u, x)=\Delta \cdot \operatorname{det}((u-Z)(x-Q)-1),
$$

where the matrices $Z$ and $Q$ are given by (3.4) and (3.5). In particular,

$$
W_{0}(x)=\Delta \cdot \operatorname{det}(x-Q) \text {. }
$$

Proof. First, we prove formula (4.2). Let $S$ and $T$ be $(n \times n)$-matrices with entries $S_{a b}=z_{b}^{a-1}$ and $T_{a b}=(a-1) z_{b}^{a-2}$, respectively. Clearly, $\operatorname{det} S=\Delta$. The entries of the matrix $S^{-1}$ are determined by the relation $P_{a}(u)=\sum_{b=1}^{n}\left(S^{-1}\right)_{a b} u^{b-1}$, so that the entries of $S^{-1} T$ are $\left(S^{-1} T\right)_{a b}=P_{a}^{\prime}\left(z_{b}\right)$.

Let $M=\operatorname{diag}\left(\mu_{1}, \ldots, \mu_{n}\right)$. Since $\partial_{x}^{k} f_{a}(x)=\left(\left(x+\mu_{a}\right) z_{a}^{k}+k z_{a}^{k-1}\right) e^{z_{a} x}$, we have

$$
W_{0}(x)=\operatorname{det}(S(x+M)+T)=\operatorname{det} S \cdot \operatorname{det}\left(x+M+S^{-1} T\right)=\Delta \cdot \operatorname{det}(x-Q) .
$$

To prove formula (4.1), set $z_{n+1}=u$. Let $\widehat{Q}$ be an $((n+1) \times(n+1))$-matrix with entries $\hat{Q}_{a b}=\left(z_{b}-z_{a}\right)^{-1}$ for $a \neq b$, and

$$
\widehat{Q}_{a a}=-\mu_{a}-\sum_{\substack{b=1 \\ b \neq a}}^{n+1} \frac{1}{z_{a}-z_{b}},
$$

where $\mu_{n+1}$ is a new variable. Set $f_{n+1}(x)=\left(x+\mu_{n+1}\right) e^{z_{n+1} x}$. Similarly to (4.2), we have

$$
e^{-\sum_{a=1}^{n+1} z_{a} x} \operatorname{Wr}\left[f_{1}(x), \ldots, f_{n+1}(x)\right]=\Delta \cdot P\left(z_{n+1}\right) \operatorname{det}(x-\widehat{Q}) .
$$

It is easy to see that

$$
\mathrm{Wr}\left[f_{1}(x), \ldots, f_{n}(x), e^{u x}\right]=\lim _{\mu_{n+1} \rightarrow \infty}\left(\mu_{n+1}^{-1} \operatorname{Wr}\left[f_{1}(x), \ldots, f_{n+1}(x)\right]\right)
$$

and

$$
\lim _{\mu_{n+1} \rightarrow \infty}\left(\mu_{n+1}^{-1} \operatorname{det}(x-\widehat{Q})\right)=\operatorname{det}\left(x-Q-(u-Z)^{-1}\right) .
$$

Then

$$
W(u, x)=\Delta \cdot P(u) \operatorname{det}\left(x-Q-(u-Z)^{-1}\right)=\Delta \cdot \operatorname{det}((u-Z)(x-Q)-1) .
$$

The lemma is proved.

The complex vector space spanned by the functions $f_{1}, \ldots, f_{n}$ is the kernel of the monic differential operator

$$
D=\partial_{x}^{n}+\sum_{a=1}^{n} C_{a}(x) \partial_{x}^{n-a} .
$$

The function $\psi(u, x)$, defined by (3.6), has the following expansion as $u \rightarrow \infty, x \rightarrow \infty$ :

$$
\psi(u, x)=1+\sum_{i=1}^{\infty} \sum_{j=1}^{\infty} \psi_{i j} u^{-j} x^{-i}
$$

Here we suppressed the arguments $z_{1}, \ldots, z_{n}, h_{1}, \ldots, h_{n}$. Set

$$
\psi_{i}(u)=\sum_{j=1}^{\infty} \psi_{i j} u^{-j}, \quad i \in \mathbb{Z}_{>0} .
$$


Lemma 4.2. We have

$$
\psi_{1}(u)=-\sum_{a=1}^{N} \frac{1}{u-z_{a}}, \quad \psi_{2}(u)=\sum_{a=1}^{N} \frac{1}{u-z_{a}}\left(-h_{a}+\sum_{b \neq a} \frac{1}{z_{a}-z_{b}}\right),
$$

and

$$
\sum_{i=1}^{\infty} \psi_{i 1} x^{-i}=-\operatorname{tr}\left((x-Q)^{-1}\right)
$$

Proof. The proof is straightforward using formulae (3.5), (3.6).

4.2. Proof of Theorem 3.2. Denote $\mathcal{D}_{\text {reg }}=P(u) \mathcal{D}$. By Theorem 3.1 in MTV2, we have

$$
\mathcal{D}_{\text {reg }}=\sum_{i=0}^{N} \sum_{a=0}^{n} A_{i a} u^{a} \partial^{i}, \quad A_{\text {ia }} \in \operatorname{End}\left(V^{\otimes n}\right),
$$

and

$$
\sum_{a=0}^{n} A_{N a} u^{a}=P(u), \quad \sum_{i=0}^{N} A_{i n} \partial^{i}=R\left(\partial_{u}\right), \quad R(x)=\prod_{i=1}^{N}\left(x-K_{i}\right) .
$$

Let $v \in \bigotimes_{a=1}^{n} V\left(z_{a}\right)$ be an eigenvector of the Bethe algebra, $A_{i a} v=\alpha_{i a} v, \alpha_{i a} \in \mathbb{C}$, for all $(i, a)$. Consider a scalar differential operator

$$
D_{v}=\sum_{i=0}^{N} \sum_{a=0}^{n} \alpha_{i a} x^{i} \partial_{x}^{a}
$$

Notice that we changed $u \mapsto \partial_{x}, \partial_{u} \mapsto x$ compared with (4.7). By Theorem 3.1 in MTV2] and Theorem 12.1.1 in [MTV4, the kernel of $D_{v}$ is generated by the functions $\left(x+\mu_{a}\right) e^{z_{a} x}, a=1, \ldots, n$, with suitable $\mu_{a} \in \mathbb{C}$. Let

$$
h_{a}=-\mu_{a}-\sum_{b \neq a} \frac{1}{z_{a}-z_{b}}, \quad a=1, \ldots, n .
$$

Lemma 4.3. We have $H_{a} v=h_{a} v$ for all $a=1, \ldots, n$.

Proof. We have $D_{v}=R(x) D$, where $D$ is given by (4.3). Then Lemma 4.1 and formulae (2.2), (2.3), (3.1) show that the eigenvalues of the operators $\Psi_{i j}$ are the numbers $\psi_{i j}$ given by (4.4): $\Psi_{i j} v=\psi_{i j} v$. The claim follows from comparing formulae (3.2) and (4.5).

By Theorem 10.5.1 in MTV4, if $K_{1}, \ldots, K_{N}$ and $z_{1}, \ldots, z_{n}$ are generic, then the Bethe algebra of $\bigotimes_{a=1}^{n} V\left(z_{a}\right)$ has an eigenbasis. Hence, by Lemmas 4.1 and 4.3 for such $K_{1}, \ldots, K_{N}, z_{1}, \ldots, z_{n}$ we have

$$
\Psi(u, x)=\psi\left(u, x, z_{1}, \ldots, z_{n}, H_{1}, \ldots, H_{n}\right) .
$$

Since both sides of this identity are meromorphic functions of $K_{1}, \ldots, K_{N}$ and $z_{1}, \ldots, z_{n}$, the identity holds true for all $K_{1}, \ldots, K_{N}, z_{1}, \ldots, z_{n}$. The theorem is proved. 


\section{§5. Bethe Algebra and functions on the Calogero-Moser space}

5.1. Calogero-Moser space $\mathcal{C}_{n}$. Let $\mathcal{M}_{n}$ be the space of complex matrices of size $n \times n$. The group $G L_{n}$ acts on $\mathcal{M}_{n} \oplus \mathcal{M}_{n}$ by conjugation, $g:(X, Y) \mapsto\left(g X g^{-1}, g Y g^{-1}\right)$. Denote $\widehat{\mathcal{F}}_{n}=\mathbb{C}\left[\mathcal{M}_{n} \oplus \mathcal{M}_{n}\right]^{G L_{n}}$.

Let $\mathcal{C}_{n} \subset \mathcal{M}_{n} \oplus \mathcal{M}_{n}$ be the subset of pairs $(X, Y)$ with the matrix $[X, Y]+1$ having rank one. The set $\mathcal{C}_{n}$ is $G L_{n}$-invariant. Let $\mathcal{I}_{n} \subset \widehat{\mathcal{F}}_{n}$ be the ideal of functions vanishing on $\mathcal{C}_{n}$. By definition, the algebra $\mathcal{F}_{n}=\widehat{\mathcal{F}}_{n} / \mathcal{I}_{n}$ is the algebra of functions on the $n$th Calogero-Moser space; see [Wi].

Consider a function

$$
\phi(u, x, X, Y)=\operatorname{det}\left(1-(u-Y)^{-1}(x-X)^{-1}\right),
$$

depending on matrices $X, Y$ and variables $u, x$. It has an expansion as $u \rightarrow \infty, x \rightarrow \infty$ :

$$
\phi(u, x, X, Y)=1+\sum_{i=1}^{\infty} \sum_{j=1}^{\infty} \phi_{i j}(X, Y) u^{-j} x^{-i}
$$

with $\phi_{i j} \in \widehat{\mathcal{F}}_{n}$ for any $(i, j)$.

Lemma 5.1 (MTV6]). The algebra $\mathcal{F}_{n}$ is generated by the images of $\phi_{i j}, i, j \in \mathbb{Z}_{>0}$.

5.2. Bethe algebra and functions on $\mathcal{C}_{n}$. In this section we treat $K_{1}, \ldots, K_{N}$ and $z_{1}, \ldots, z_{n}$ as variables. Set

$$
\mathcal{E}_{N, n}=\operatorname{End}\left(V^{\otimes n}\right) \otimes \mathbb{C}\left[K_{1}, \ldots, K_{N}, z_{1}, \ldots, z_{n}\right] .
$$

We identify the algebras $\operatorname{End}\left(V^{\otimes n}\right)$ and $\mathbb{C}\left[K_{1}, \ldots, K_{N}, z_{1}, \ldots, z_{n}\right]$ with the respective subalgebras $\operatorname{End}\left(V^{\otimes n}\right) \otimes 1$ and $1 \otimes \mathbb{C}\left[K_{1}, \ldots, K_{N}, z_{1}, \ldots, z_{n}\right]$ of $\mathcal{E}_{N, n}$.

The operators $B_{i j}$ and $\Psi_{i j}$, defined in $\oint 3$, depend on $K_{1}, \ldots, K_{N}, z_{1}, \ldots, z_{n}$ polynomially, so we view them as elements of $\mathcal{E}_{N, n}$. Denote by $\mathcal{B}_{N, n}$ the unital subalgebra of $\mathcal{E}_{N, n}$ generated by $B_{i j}, i=1, \ldots, N, j \in \mathbb{Z}_{\geq 0}$.

Lemma 5.2. The algebra $\mathcal{B}_{N, n}$ is generated by $\Psi_{i j}, i=1, \ldots, N, j \in \mathbb{Z}_{>0}$, and symmetric polynomials in $K_{1}, \ldots, K_{N}$.

Proof. By formula (2.1), we have

$$
x^{N}+\sum_{i=1}^{N} B_{i 0} x^{N-i}=\prod_{i=1}^{N}\left(x-K_{i}\right),
$$

so that symmetric polynomials in $K_{1}, \ldots, K_{N}$ belong to $\mathcal{B}_{N, n}$. Formula (2.2) yields

$$
\left(x^{N}+\sum_{i=1}^{N} \sum_{j=0}^{\infty} B_{i j} u^{-j} x^{N-i}\right) \prod_{i=1}^{N} \frac{1}{x-K_{i}}=1+\sum_{i=1}^{\infty} \sum_{i=1}^{\infty} \Psi_{i j} u^{-j} x^{-i}
$$

Therefore, the elements $\Psi_{i j}$ are linear combinations of the elements $B_{i j}$ with coefficients being symmetric polynomials in $K_{1}, \ldots, K_{N}$, and vice versa. That proves the claim.

Let $Z, Q$ be the matrices given by (3.4), (3.5). For any $f \in \widehat{\mathcal{F}}_{n}$, define a function $\bar{f}$ of the variables $z_{1}, \ldots, z_{n}, h_{1}, \ldots, h_{n}$ by the formula

$$
\bar{f}\left(z_{1}, \ldots, z_{n}, h_{1}, \ldots, h_{n}\right)=f(Q, Z) .
$$

Lemma 5.3. The function $\bar{f}$ depends only on the image of $f$ in $\mathcal{F}_{n}$.

Proof. Since the matrix $[Q, Z]+1$ has rank one, the pair $(Q, Z)$ belongs to $\mathcal{C}_{n}$.

Theorem 5.4. For any $f \in \widehat{\mathcal{F}}_{n}$, we have $\bar{f}\left(z_{1}, \ldots, z_{n}, H_{1}, \ldots, H_{n}\right) \in \mathcal{B}_{N, n}$. In particular, $f\left(z_{1}, \ldots, z_{n}, H_{1}, \ldots, H_{n}\right)$ is a polynomial in $z_{1}, \ldots, z_{n}$. 
Proof. By Lemmas 5.3 and 5.1, it suffices to prove the claim for the functions $\phi_{i j}(X, Y)$. Since $\bar{\phi}_{i j}=\psi_{i j}$ by (5.1), (5.2), (3.6), and (4.4), and $\psi_{i j}\left(z_{1}, \ldots, z_{n}, H_{1}, \ldots, H_{n}\right)=\Psi_{i j}$ by Theorem 3.2, the statement follows from Lemma 5.2.

Example. Let $N=n=2$. Then $Z=\operatorname{diag}\left(z_{1}, z_{2}\right)$,

$$
\begin{aligned}
Q & =\left(\begin{array}{cc}
h_{1} & \left(z_{2}-z_{1}\right)^{-1} \\
\left.z_{1}-z_{2}\right)^{-1} & h_{2}
\end{array}\right), \\
H_{1} & =K_{1} e_{11}^{(1)}+K_{2} e_{22}^{(1)}+\frac{\Omega}{z_{1}-z_{2}}, \\
H_{2} & =K_{1} e_{11}^{(2)}+K_{2} e_{22}^{(2)}+\frac{\Omega}{z_{2}-z_{1}}, \\
\Omega & =e_{11}^{(1)} e_{11}^{(2)}+e_{12}^{(1)} e_{21}^{(2)}+e_{21}^{(1)} e_{12}^{(2)}+e_{22}^{(1)} e_{22}^{(2)} .
\end{aligned}
$$

Let $f(X, Y)=\operatorname{tr}\left(X^{2}\right)$. Then $\bar{f}\left(z_{1}, z_{2}, H_{1}, H_{2}\right)=H_{1}^{2}+H_{2}^{2}-2\left(z_{1}-z_{2}\right)^{-2}$ is a polynomial in $z_{1}, z_{2}$.

Remark. It is known that $\hat{\mathcal{F}}_{n}$ is spanned by the functions

$$
\operatorname{tr}\left(X^{m_{1}} Y^{m_{2}} X^{m_{3}} Y^{m_{4}} \cdots\right),
$$

where $m_{1}, m_{2}, \ldots$ are nonnegative integers; see [W].

Theorems 3.2 and 5.4 show that the assignment $\gamma: f \mapsto \bar{f}\left(z_{1}, \ldots, z_{n}, H_{1}, \ldots, H_{n}\right)$ defines an algebra homomorphism $\hat{\mathcal{F}}_{n} \rightarrow \mathcal{B}_{N, n}$ that sends $\phi_{i j}$ to $\Psi_{i j}$. By Lemma 5.3, this homomorphism factors through $\mathcal{F}_{n}$. By Lemma 5.2, the images of $\hat{\mathcal{F}}_{n}$ tensored with the algebra of symmetric polynomials in $K_{1}, \ldots, K_{N}$ generate $\mathcal{B}_{N, n}$.

We showed in MTV6] that for $n=N$, the homomorphism $\gamma$ induces an isomorphism of $\mathcal{F}_{N}$ with the quotient of $\mathcal{B}_{N, N}$ by the relations

$$
\Psi_{i 1}=-\sum_{j=1}^{N} K_{j}^{i-1}, \quad i \in \mathbb{Z}_{>0} .
$$

In other words, let

$$
\left(V^{\otimes N}\right)_{\mathbf{1}}=\left\{v \in V^{\otimes N} \mid \sum_{a=1}^{N} e_{i i}^{(a)} v=v, i=1, \ldots, N\right\} .
$$

Each element of $\mathcal{B}_{N, N}$ induces an element of

$$
\operatorname{End}\left(\left(V^{\otimes N}\right)_{1}\right) \otimes \mathbb{C}\left[K_{1}, \ldots, K_{N}, z_{1}, \ldots, z_{N}\right] .
$$

Then $\mathcal{F}_{N}$ is isomorphic to the image of $\mathcal{B}_{N, N}$ in

$$
\operatorname{End}\left(\left(V^{\otimes N}\right)_{1}\right) \otimes \mathbb{C}\left[K_{1}, \ldots, K_{N}, z_{1}, \ldots, z_{N}\right] .
$$

\section{REFERENCES}

[B] H. M. Babujian, Off-shell Bethe ansatz equations and $N$-point correlators in the $\mathrm{SU}(2)$ WZNW theory, J. Phys. A 26 (1993), no. 23, 6981-6990. MR1253889 (95a:82028)

[CT] A. Chervov and D. Talalaev, Quantum spectral curves, quantum integrable systems and the geometric Langlands correspondence, Preprint, 2006, 54 pp.; hep-th/0604128

[FFR] B. Feigin, E. Frenkel, and N. Reshetikhin, Gaudin model, Bethe ansatz and critical level, Comm. Math. Phys. 166 (1994), no. 1, 27-62. MR1309540 (96e:82012)

[FMTV] G. Felder, Y. Markov, V. Tarasov, and A. Varchenko, Differential equations compatible with KZ equations, Math. Phys. Anal. Geom. 3 (2000), no. 2, 139-177. MR1797943 (2002e:17032)

[G1] M. Gaudin, Diagonalisation d'une classe d'Hamiltoniens de spin, J. Physique 37 (1976), no. 10, 1089-1098. MR0421442 
[G2] La fonction d'onde de Bethe, Collection du Commissariat à l'Énergie Atomique: Sér. Sci., Masson, Paris, 1983. MR0693905 (85h:82001)

[KS] P. P. Kulish and E. K. Sklyanin, Quantum spectral transform method. Recent developments, Lecture Notes in Phys., vol. 151, Springer, Berlin-New York, 1982, pp. 61-119. MR0671263 $(84 \mathrm{~m}: 81114)$

[MTV1] E. Mukhin, V. Tarasov, and A. Varchenko, Bethe eigenvectors of higher transfer matrices, J. Stat. Mech. Theory Exp. 2006, no. 8, P08002, 44 pp. MR2249767 (2007h:82021)

[MTV2] _ A generalization of the Capelli identity, Algebra, Arithmetic, and Geometry: in Honor of Yu. I. Manin. Vol. II, Progr. Math., vol. 270, Birkhäuser Boston, Inc., Boston, MA, 2009, pp. 383-398. MR2641196

[MTV3] _ Schubert calculus and representations of the general linear group, J. Amer. Math. Soc. 22 (2009), no. 4, 909-940. MR2525775

[MTV4]_ Generating operator of XXX or Gaudin transfer matrices has quasi-exponential kernel, SIGMA Symmetry Integrability Geom. Methods Appl. 3 (2007), 060, 31 pp. MR2299861 (2008a:81095)

[MTV5] , Spaces of quasi-exponentials and representations of $\mathfrak{g l}_{N}$, J. Phys. A 41 (2008), 194017, 28 pp. MR 2452191 (2010g;17010)

[MTV6] _ Bethe algebra, Calogero-Moser space and Cherednik algebra, Preprint, 2009, 24 pp., arXiv:0906.5185

[RV] N. Reshetikhin and A. Varchenko, Quasiclassical asymptotics of solutions to the KZ equations, Geometry, Topology, \& Physics, Conf. Proc. Lecture Notes Geom. Topology, IV, Int. Press, Cambridge, MA, 1995, pp. 293-322. MR1358621 (96j:32025)

[SV] V. Schechtman and A. Varchenko, Arrangements of hyperplanes and Lie algebra homology, Invent. Math. 106 (1991), no. 1, 139-194. MR.1123378 (93b:17067)

[T] D. Talalaev, Quantization of the Gaudin system, Preprint, 2004, 19 pp., hep-th/0404153

[W] H. Weyl, The classical groups. Their invariants and representations, Princeton Univ. Press, Princeton, NJ, 1939. MR0000255 (1:5g)

[Wi] G. Wilson, Collisions of Calogero-Moser particles and an adelic Grassmannian, Invent. Math. 133 (1998), 1-41. MR:1626461 (99f:58107)

Department of Mathematical Sciences, Indiana University - Purdue University IndianapoLis, 402 North Blackford St., Indianapolis, Indiana 46202-3216

E-mail address: mukhin@math.iupui.edu

Department of Mathematical Sciences, Indiana University - Purdue University IndianapoLis, 402 North Blackford St., Indianapolis, Indiana 46202-3216

E-mail address: vt@math.iupui.edu

Current address: St. Petersburg Branch, Steklov Mathematical Institute, Russian Academy of Sciences, Fontanka 27, St. Petersburg 191023, Russia

E-mail address: vt@pdmi.ras.ru

Department of Mathematics, University of North Carolina at Chapel Hill, Chapel Hill, North CAROLINA 27599-325

E-mail address: anv@email.unc.edu

Received $15 / \mathrm{NOV} / 2009$

Originally published in English 